\title{
El uso de los fractales para potenciar el desarrollo del pensamiento algebraico-variacional a través del software cabrí “Del pensamiento numérico al pensamiento Algebraico-Variacional"1
}

\author{
The use of fractals to enhance the development of algebraic \\ thinking-variational through Cabri "From thought to \\ thought Algebraic-numerical variational" \\ O uso de fractais para melhorar o desenvolvimento do \\ pensamento algébrico-variacional através Cabri "de pensamento \\ a pensamento variacional Algébrica-numérico"
}

Recibido: mayo de 2013

Aceptado: agosto de 2013
Jose Francisco Puerto Monterroza ${ }^{2}$

\section{Resumen}

El propósito de este taller es mostrar como con el uso del cabrí, y la construcción de algunos fractales (conjunto de Cantor, el triángulo de Sierpinski y el copo de nieve de Von Koch) se pueden identificar patrones numéricos y/o geométricos. Estas actividades preparan a los estudiantes para la construcción de la expresión algebraica a través de la formulación verbal de una regla recursiva que muestre cómo construir los términos siguientes a partir de los precedentes y el hallazgo de un patrón que los guíe más o menos directamente a la expresión algebraica. Esta es una forma muy apropiada de preparar el aprendizaje significativo y comprensivo de los sistemas algebraicos y su manejo simbólico para mejorar el proceso de transición de la Aritmética al Algebra.

Palabras clave: Patrones numéricos; generalización; sistemas algebraicos; expresión algebraica; matemáticas escolares; geometría; fractales; aula; recursos didácticos; recursos informáticos; software.

\begin{abstract}
The purpose of this workshop is to show how to use Cabri, and the construction of some fractals (Cantor set, the Sierpinski triangle and snowflake Von Koch) number patterns can be identified and / or geometric. These activities prepare students for the construction of the algebraic expression through verbal formulation of recursive rule that shows how to build the following terms from the preceding and the finding of a pattern to guide more or less directly to the algebraic expression. This is a very appropriate way to prepare meaningful learning and understanding algebraic systems and symbolic management to improve the transition from arithmetic to algebra.
\end{abstract}

1 Artículo de Investigación.

2 Universidad De Sucre Sincelejo-Sucre, Colombia. Contacto: jopuermon@gmail.com 
Keywords: Number Patterns; generalization algebraic systems; algebraic expression; school mathematics, geometry, fractals, classroom teaching resources, computing resources, software.

\section{Resumo}

O objetivo deste workshop é mostrar como usar o Cabri, ea construção de alguns fractais (Cantor set, o triângulo de Sierpinski e floco de neve Von Koch) padrões de número podem ser identificados e / ou geométrica. Essas atividades preparar os alunos para a construção da expressão algébrica através da formulação verbal de regra recursiva que mostra como construir os seguintes termos da anterior eo achado de um padrão para orientar mais ou menos diretamente à expressão algébrica. Esta é uma maneira muito apropriada para se preparar de aprendizagem e compreensão significativa de sistemas algébricos e gestão simbólica para melhorar a transição da aritmética para a álgebra.

Palavras-chave: padrões numéricos, sistemas de generalização algébricas; expressão algébrica, a matemática escolar, geometria, fractais, recursos de ensino em sala de aula, recursos de computação, de software.

\section{Introducción}

"La exposición repetida de construcciones de formulas, como expresiones que explicitan un patrón de variación, ayuda a los estudiantes a comprender la sintaxis de las expresiones algebraicas que aparecerán después en el estudio del algebra”. Demana (1990).

Diferentes investigaciones realizadas en los últimos años (TIMSS, PISA, etc) develan las dificultades que presentan los estudiantes de los primeros niveles de la educación secundaria cuando se enfrentan a problemas algebraico, manipulación de expresiones algebraicas y significación de éstas, interpretación de información dada en distintos lenguajes (gráficos, tabulares, algebraicos) y solución de problemas de cambio y variación, entre otros. Esto se debe quizás a que el currículo durante los primeros grados está basado solamente en el estudio de la aritmética, y que tópicos como el Algebra, que podrían ayudar a los estudiantes a desarrollar destrezas de pensamiento como: observar, analizar, conjeturar, generalizar, etc., son reservados para ser estudiados en los grados superiores. Además, la aritmética frecuentemente se enfoca en los resultados de los procesos de cálculo más que en los aspectos relacionales y estructurales, oponiéndose así al reconocimiento de las reglas del álgebra, que constituyen expresiones que expresan generalidades, donde los patrones que se observan aparecen en las mismas colecciones de números, $\mathrm{y}$ en las operaciones comunes que se hacen con estos números o como modelos que describen situaciones.

El hecho de que el álgebra pueda ser vista como la formulación y manipulación de proposiciones generales sobre los números, hace que la experiencia previa que el estudiante ha tenido con la estructura de expresiones numéricas en la escuela, tenga efecto sobre la habilidad para asignarle sentido a esta. Por esto el Consejo Nacional de Profesores de Matemáticas de Estados Unidos (NCTM), establece que se deben propiciarse actividades que involucren la generalización de patrones numéricos para modelar, representar o describir patrones físicos, regularidades y patrones que se hayan observado. Estas exploraciones informales de conceptos algebraicos deben contribuir a que el estudiante adquiera confianza en su propia capacidad de abstraer relaciones a partir de información contextual y de utilizar toda una gama de representaciones para describir dichas relaciones. Cuando los estudiantes elaboran gráficas, tablas de datos, expresiones, ecuaciones o descripciones verbales 
para representar una relación simple, descubren que representaciones diferentes dan lugar a diferentes interpretaciones de una situación.

Esta propuesta se fundamenta en lo descritos anteriormente y se plantean actividades de generalización de patrones numéricos, geométricos y de leyes y reglas de tipo natural o social que rigen los números y las figuras; se involucra la visualización, exploración y manipulación de los números y las figuras en los cuales se basa el proceso de generalización como lo propone Mason (1992). Estas actividades preparan a los estudiantes para la construcción de la expresión algebraica a través de la formulación verbal de una regla recursiva que muestre cómo construir los términos siguientes a partir de los precedentes y el hallazgo de un patrón que los guíe más o menos directamente a la expresión algebraica. De esta manera, el pensamiento algebraico surge como generalización del trabajo aritmético con modelos numéricos en situaciones de variación.

\section{Marco teórico}

La generalidad es un aspecto central en la actividad matemática, a todo nivel, $y$, a la cual se puede retornar una y otra vez, cualquiera que sea el tema particular de discusión. Las matemáticas comprenden muchas generalizaciones, ya sea que tomen forma de métodos, procedimientos, o de fórmulas, y estas pueden ser vistas como originándose de la misma manera que las propias generalizaciones de los patrones, hechas por los alumnos.

La generalización es uno de los procesos que ocurren en cualquier nivel del pensamiento matemático y que está incluido en uno más global, el proceso de abstraer, "To generalize is to derive or induce from particulars, to identify commonalities, to expand domains of validity" (Dreyfus, 1991, p. 35). La generalización es fundamental para el desarrollo del pensamiento matemático y algebraico, es base de la abstracción (Mason, 1985), es indudable entonces la importancia de su tratamiento. Está relacionada con otros procesos propios de la actividad matemática, que podrían denominarse más particulares como: inducir, observar, descomponer, hacer analogías e identificar características comunes. Y debe pasar por varias etapas; a saber: La percepción de un patrón, la expresión del patrón, el registro del patrón y la prueba de validez del patrón (Mason, Socas, Sessa, Butto y Rojano).

Por otra parte las reglas del álgebra constituyen expresiones que expresan generalidades, pero los patrones que se observan aparecen en las mismas colecciones de números, y en las operaciones comunes que se hacen con estos números o como modelos que describen situaciones. El álgebra es el lenguaje con que se expresa dicha generalidad. Para aprender el lenguaje del álgebra es necesario tener algo que decir, se debe percibir algún patrón o regularidad y luego tratar de expresarlo en forma sucinta, para poder comunicarlo a alguien. (Rutas hacia el álgebra. John Mason y otros, 1999). Es así como el mayor reto en la enseñanza del álgebra es promover la percepción de la "generalidad" que esta detrás de los símbolos, para lo cual es necesario ampliar la notación del lenguaje aritmético y utilizar las propiedades características de los sistemas numéricos. De esta manera, el pensamiento algebraico surge como generalización del trabajo aritmético con modelos numéricos en situaciones de variación.

Una manera propicia de experimentar con procesos de generalización y búsqueda de patrones, entre otros, es aprovechar las posibilidades que brindan la incorporación de las TICs en el aula y muy especialmente el software de geometría dinámica CABRI, por que permite: el descubrimiento, la experimentación, la exploración, la construcción de modelos, la formulación de hipótesis, la demostración, la responsabilidad, la creatividad, el análisis, el trabajo colaborativo, en fin la actividad del estudiante sobre el objeto-conocimiento desde diferentes aristas (Cervantes \& Viquez, 1994, 144), es decir, ver la matemática de una manera mas activa y dinámica: la matemática experimental.

Este taller se fundamenta en este software y en la hoy naciente GEOMETRÍA FRACTAL, que es una poderosa herramienta que permite modelar fenómenos impredecibles y fascinantes de la naturaleza y que fue dada a conocer al mundo en los años setenta por Benoît Mandelbrot. El término fractal 
fue acuñado por el y hace referencia a la idea de "partido" o "fracturado". Como característica fundamental se considera la autosemejanza o autosimilaridad de su forma, la reiteración o iteración en la formación de su modelo, la dimensión que intenta describir su tamaño o densidad y el concepto de atractor para caracterizar la figura cuando el proceso de iteración tiende al infinito. Esta idea es suficiente para pensar en figuras que, por ejemplo, tienen área finita y perímetro infinito. Quizás algunos conjuntos ya clásicos puedan resultar familiares, y que podrían servir de ejemplos bastante sencillos para estas características (como el conjunto de Cantor, el triángulo de Sierpinski, o la curva de Koch). Cabe indicar que este trabajo se centra mas en las regularidades numéricas que se presentan entre los elementos constitutivos del fractal, que en la construcción formal de los conceptos fractales.

El trabajo con actividades sobre reconocimiento de patrones y su generalización, proporciona la oportunidad de acordar nuevas formas de comunicación en las que prevalece y se le da sentido al lenguaje algebraico como una forma sucinta para expresar conjeturas, y someterlas a verificación y refutación. Las actividades planteadas sobre generalización, posibilitan el desarrollo de habilidades como la predicción y la sistematización, las cuales se deben explicitar, para propiciar la discusión sobre su importancia no solo en las matemáticas sino en situaciones cotidianas.

El propósito de este proyecto de aula es mostrar como con el uso del Cabrí, y a través de la manipulación de objetos geométricos y la identificación de regularidades numéricas, se puede mejorar el tratamiento del proceso de transición de la Aritmética al Algebra, trabajando dos aspectos centrales: el desarrollo del Pensamiento Numérico y el Pensamiento Algebraico.

\section{Metodologia}

La metodología a utilizar en este taller esta fundamentada en el uso del software de geometría dinámica Cabrí, y la inmersión en el fascinante mundo de los fractales que permite a través de construcciones geométricas modelar y explorar problemas que conllevan a desarrollar en los alumnos los pensamientos matemáticos. Se hace la construcción de algunos fractales como el conjunto de Cantor, el triángulo de Sierpinski y el copo de nieve de Von Koch y se identifican las regularidades numéricas que se presentan entre los elementos constitutivos del fractal, se registran estos resultados para así poder determinar su expresión algebraica. Esta es una forma muy apropiada de preparar el aprendizaje significativo y comprensivo de los sistemas algebraicos y su manejo simbólico.

\section{Objetivo general}

- Favorecer un acercamiento significativo a conceptos fundamentales del álgebra como expresiones algebraicas y ecuaciones, desde actividades funcionales y de generalización.

\section{Objetivos específicos}

- Fomentar el uso de las TICs en el desarrollo de los procesos de aula.

- Propiciar en los estudiantes el desarrollo de competencias para: observar, medir, valorar, analizar e interpretar situaciones numéricas.

- Desarrollar en los estudiantes competencias para: ver relaciones y establecer conexiones, hacer predicciones y generalizaciones, hasta llegar a la modelización y la formalización de leyes

- Fomentar el desarrollo del razonamiento inductivo-deductivo.

- Propiciar el desarrollo de la competencia comunicativa.

- Crear espacios para el trabajo cooperativo.

- Mejorar los procesos de enseñanza y de aprendizaje de las matemáticas.

\section{Resultados que se esperan obtener}

Afianzar la comprensión de las operaciones matemáticas básicas.

El desarrollo de valores como: la autoestima, la responsabilidad, la autonomía, la solidaridad, la creatividad y el emprendimiento. 
La ceración de nuevas ideas a partir de lo concreto para interiorizar conceptos abstractos.

El desarrollo de competencias para la identificación de regularidades numéricas para su generalización y modelación.

Familiarizar al estudiante con el manejo del lenguaje gráfico-tabular-algebraico.

Desarrollar en los alumnos habilidades de orden superior como explorar, conjeturar, razonar, reflexionar y comunicar matemáticamente, así como habilidad para usar efectivamente sus habilidades cognitivas y metacognitivas en la solución de problemas rutinarios.

Minimizar las deficiencias que se presentan en el proceso de transición de la aritmética al algebra.

Fomentar el trabajo cooperativo.

\section{Estandares de calidad a desarrollar}

\section{Pensamiento numérico y sistemas numéricos}

Reconocer significados del número en diferentes contextos (medición, conteo, comparación, codificación, localización, etc).

Identificar regularidades y patrones numéricos, las propiedades de los números, sus relaciones y operaciones utilizando calculadoras o computador.

\section{Pensamiento espacial y sistemas geométricos}

Diferenciar atributos y propiedades de objetos bidimensionales y tridimensionales.

Hacer conjeturas y verificar los resultados de aplicar transformaciones a figuras en el plano.

\section{Pensamiento variacional y sistemas algebraicos y analíticos}

Reconocer y describir regularidades y patrones en distintos contextos (numérico, geométrico, etc).

Predecir patrones de variación en una secuencia numérica, geométrica o gráfica.

Describir y representar situaciones de variación relacionando diferentes representaciones (diagramas, expresiones verbales generalizadas y tablas).

Reconocer el conjunto de valores de una variable en situaciones concretas de cambio (variación).

Usar procesos inductivos y lenguaje algebraico para verificar conjeturas.

Modelar situaciones de variación con funciones polinómicas

\section{Referencias}

Briggs, J. (1994). Espejo y Reflejo: Del Orden al Caos. Barcelona: Gedisa.

Guzmán, M. (1993). Estructuras Fractales y sus Aplicaciones. Barcelona: Labor.

García, A. (1995). Nuevas Tecnologías y Enseñanza de Las Matemáticas. Madrid: Síntesis.

Ministerio de Educación Nacional (1999). Nuevas Tecnologías y Currículo de Matemáticas. Bogotá: Punto Exe editores.

Mason J. (1999). Rutas hacia el álgebra .Traducción por Cecilia Agudelo Valderrama. Universidad Pedagógica y Tecnológica de Colombia

Ministerio de Educación Nacional. (2003). Estándares Básicos de calidad - Matemáticas.

Ministerio de Educación Nacional. (1998). Lineamientos curriculares - Matemáticas.

Rosillo, N. (1997). Fractales con el Miniordenador TI-92. Madrid: Faster

Soca, M. M., (1997): “Dificultades, obstáculos y errores en el aprendizaje de las Matemáticas en la Educación Secundaria, cap. 5, pp. 113-141" en L. Rico y otros: La educación Matemática en la Enseñanza Secundaria. Ed. Horsori (en prensa)

Soca, M. M., y otros (1989). Iniciación al Algebra. Síntesis. Madrid.

Soca, M. M., y Palarea, M. M. (1987). Las fuentes de significado, los sistemas de representación y errores en el algebra escolar. Uno, núm14, pp. 7-24. Barcelona 